\title{
Do you really know what Periodontal Disease is?
}

\author{
Jose Ricardo Kina* \\ Department of Periodontics, UNESP Universidade Estadual Paulista Júlio de Mesquita Filho, Brazil
}

Received: 門January 18, 2018; Published: 畊January 25, 2018

*Corresponding author: Jose Ricardo Kina, Department of Periodontics, UNESP, Barzil

\section{Introduction}

The etiology includes the sum of evidences related to the causes of a disease. Majority of all forms of periodontal diseases, are considered as microorganisms-induced dependent. However, it is improbable that bacteria alone cause periodontal disease. The etiological concept of the inflammatory periodontal disease is an exceedingly complex interaction of bacteria and predisposing risk factors. The predisposing risk factor may be an inherent characteristic associated with an increased rate of a subsequently occurring disease, but does not necessarily cause the disease. In periodontal disease, the predisposing risk factors may be defined as local environmental factors, behavioral factors in nature and systemic factors, which may be responsible in providing an ideal environment for bacterial colonization and/or fragility in a determinate tooth or teeth and adjacent periodontal tissues and/ or interference in the inflammatory process. Local environmental factor may interfere in the fragile equilibrium of the gingival sulcus defense by favoring microbial colonization and growth or/and altering the local susceptibility of the periodontal tissues to be damaged by the bacterial onslaught.

When predisposing risk systemic factors affect the individual, a deficient interaction of the bacteria with cells of the inflammatory process may occur, inducing an incomplete defensive process, leading to the periodontal destruction. Periodontal disease could be considered as sequel of the inflammatory reaction, which must be always active, protecting individual against infection and possible septicemia, by bacteria present in the gingival sulcus, a critical area where junctional epithelium is an exclusive and fragile structure, separating connective tissue from an infected humid and warm oral environment. Periodontitis a protective inflammatory mechanism, begins with microbial challenge, which induce a hostmediate response and destruction of periodontal tissue, caused by bursts of clastic cell activity, triggered by hyperactivated or primed polymorphonuclear leukocytes and factors generated during the inflammatory acute phase, such as eicosanoids and various proteins as enzymes that cause damage and rupture of the periodontium, as ulceration of the junctional epithelium, loss of connective tissue and alveolar bone, causing apical migration of the junctional epithelium, promoting periodontal pocket establishment.

Periodontal pocket development is the most important clinical and pathologic alteration associated with inflammatory periodontal disease and also may be considered as a local predisposing risk factor for periodontal disease progression, by generating an anaerobic environment to be contaminated as a result of repeated infection by the various species or combination of the species as exogenous anaerobic and facultative bacteria (periodontopathogenic bacteria). These putative periodontal pathogens and their products may induce substantial pathological alterations, essentially in root surface exposed to the contaminated periodontal pocket. On the other side, due bacterial approximation to the ulcerated pocket epithelium, infected periodontal pocket also could be an infectious focus linked to the various systemic disorders, probably led by anachoresis, a process associated with dissemination of the microorganisms or/and toxics products into blood stream, assisting or causing infection in the various vital organs. In addition, the destruction produced during the periodontal disease progression, may present similar characteristics, but the association among the bacteria and the various predisposing risk factors, may be distinct and inherent to each person, depending on the host's susceptibility, which does not always present an identical susceptibility to the various local and/or general predisposing risk factors.

All types of periodontal disease are multifactorial diseases, which progress through successive destructive acute phases, always interposed, by reparative chronic phases. The indication of the periodontal disease reparative phase is possible to find in untreated periodontal pocket, as cementum and the gingivalattached connective tissue zone, separating the apical end of the ulcerated periodontal pocket epithelium from the underlying destructed alveolar bone which always presents a repaired cortical bone at a range of levels protecting the cancellous bone. In untreated periodontal disease, the gingival-attached connective tissue zone should be destructed, but they arise most often in untreated periodontal pockets. After each successive destructive 
acute phase, variations in the quantity and quality of the etiological agents and the predisposing risk factors would arise. However, periodontal disease always ceases the brief acute destructive phase beginning in the sequence a long chronic reparative phase.

This fact demonstrates that, etiological agents and predisposing risk factors during the periodontal disease progression, acquired better quality and quantity, but could not be able to maintain the periodontal disease destructive phase activity all the time. Probably, the periodontal disease initiates and progress when at a given time, a specific temporary fragility, inherent for each individual, assists involved etiological agents in initiating the destructive acute phase to establish the progression of the periodontal disease. Then periodontal disease is a complex defensive mechanism which avoids penetration of bacteria into the periodontal tissues. However as a side effect this mechanism of protection induces periodontal tissues destruction that is considered as periodontal disease. Based on these considerations we can reflect asking some questions:

i. Periodontal disease is a disease? ii. Is it possible to prevent periodontal disease only through biofilm control?

iii. What is the best method to diagnostic the destructive phase of the periodontal disease?

iv. What is the best time to treat the periodontal disease: in the stable and reparative chronic phase or in the acute destructive phase?

v. Is it possible treating periodontal disease only by applying conventional periodontal therapy as scaling and root planning?

vi. sIs it possible to induce experimental periodontal disease only by seeding periodontopathogenic bacteria in a group of teeth without a predisposing risk factor as a ligature around the teeth?

These are only some of the many questions that we can still ask about the periodontal disease. Do general dentists really know what periodontal disease is and really know how to treat it?

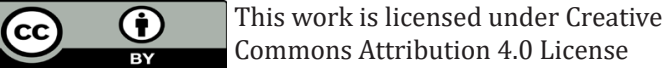

Submission Link: $\quad$ Submit Article

DOI: 10.32474/MADOHC.2018.01.000102

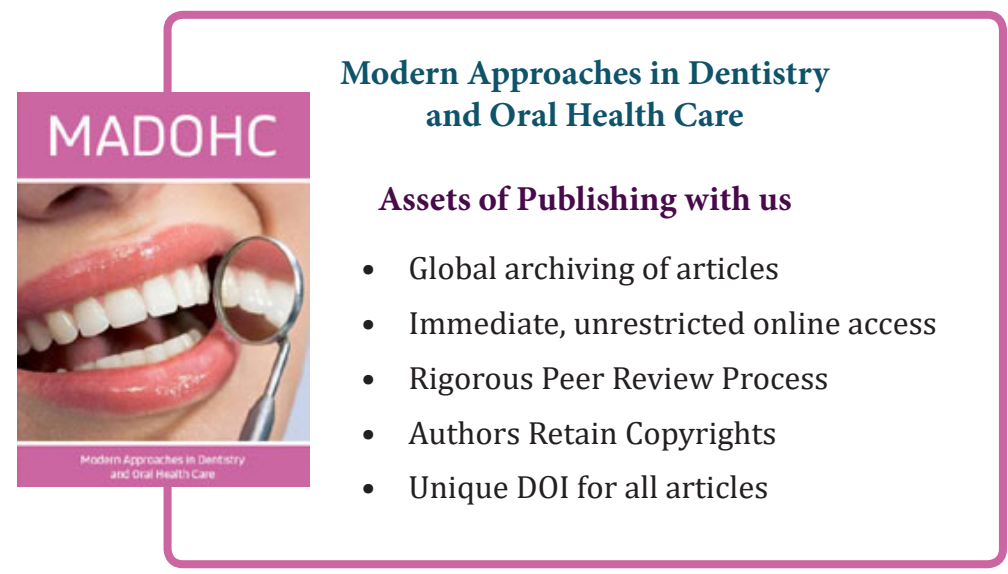

